Ein erweitertes Modell zur vergleichenden Umweltleistungsbeurteilung von Unternehmen

\section{Stimmt die Chemie?}

\section{Der Vergleich der Umweltleistung von nach ISO 14001 zertifizierten Unter- nehmen ist schwierig. Hauptproblem ist, dass es keine einheitlichen Kriterien zur vergleichenden Messung gibt. Nun liegt ein Modell zum Vergleich vor, das die Leistungsbeurteilung um soziale sowie Zukunftsaspekte erweitert und in verschiedenen Unternehmen in Deutschland und Brasilien erprobt wurde.}

$\mathrm{W}$ Von A. Grothe-Senf und B. Frank eltweit sind inzwischen circa 40.000 Unternehmen nach der DIN EN ISO 14001 - Norm zum Aufbau eines Umweltmanagementsystems zertifiziert. Sie ist gerade für Großunternehmen eine ,freiwillige“ Pflicht, wenn diese international wettbewerbsfähig bleiben wollen. Die Verpflichtung zu einer kontinuierlichen Verbesserung des Umweltmanagementsystems und der Umweltleistung der Organisation stellt das eigentliche Ziel der ISO Norm 14001 dar. Nur bleibt dabei höchst unklar, was darunter zu verstehen ist.

\section{- Interpretationsspielräume für Unternehmen}

Die Norm enthält in erster Linie die zum konkreten Aufbau eines Umweltmanagementsystems notwendigen Elemente. Die Norm und der dazu gehörende Leitfaden der ISO 14004 geben keinerlei Hinweise darauf, was die Verbesserung der Umweltleistung konkret umfasst und wie sie jeweils zu interpretieren ist. Allein die Definition von umweltorientierter Leistung als: ,messbare Ergebnisse des Umweltmanagementsystems einer Organisation in Bezug auf die Beherrschung ihrer Umweltaspekte" zeigt den Freiraum der Interpretation und macht deutlich, dass sich hier die Unternehmen bezüglich der Umweltleistung nicht vergleichen lassen (1).

Die DIN ISO Norm 14031 von 1999 zur Umweltleistungsbewertung könnte einen neuen Impuls in Richtung eines einheitlichen Verständnisses geben. Sie ist jedoch keine zertifizierbare Norm, sondern stellt eine Ergänzung zu den anderen Normen dar, indem sie unter Umweltleistung nicht nur die operativen Leistungskennzahlen versteht, sondern auch die Managementaktivitäten einer Organisation und die Indikatoren der lokalen/regionalen Umweltqualität mit einbezieht (2). Es ist damit nicht verwunderlich, dass schon zwifestzustellen sind. schen den in Deutschland zertifizierten Unternehmen große Unterschiede im Verständnis und damit auch in der Qualität der Umweltleistung

Weitet man den Vergleich auf andere Länder aus, so verstärkt sich dieser Eindruck. So wird in den USA der Aspekt des „,compliance audits“ sehr viel mehr gewichtet als beispielsweise in Brasilien. Der ökonomisch wie ökologisch sinnvolle kontinuierliche Verbesserungsprozess ist vielleicht zum Teil gar keiner, weil die Kennzahlen nicht vergleichbar sind, andere Bemessungsgrundlagen zur Unvergleichbarkeit führen, Umweltaspekte verlagert oder nicht erfasst worden sind. Eine Bewertung der Verbesserung des betrieblichen Umweltschutzes ist bisher, gemessen an der tatsächlichen Verbesserung, auch - wenn überhaupt - nur in Einzelfällen möglich. Der Einfluss der Betriebe auf die Umwelt ist oft nicht direkt nach-

zuweisen. Das liegt zum einen daran, dass sich die Wirkungen mit denen anderer Akteure vermischen oder sich die Übertragungswege aufgrund natürlicher Bedingungen wie der Wetterlage schwer nachvollziehen lassen. Zum anderen liegt es aber auch daran, dass in kleinen und mittleren Unternehmen die notwendigen Leistungsdaten nicht ermittelt werden bzw. nicht ermittelbar sind. Die Bewertung auf der Basis der Verbesserung des Umweltmanagements erweist sich als zu oberflächlich, die Bewertung der tatsächlichen Umweltwirkung als zu komplex.

Vor diesem Hintergrund wird ein enormer Forschungsbedarf deutlich, dem in einem deutschbrasilianischen Forschungsprojekt zum internationalen Vergleich der Umweltleistung von Unternehmen nachgegangen wurde (3). Besondere Aufmerksamkeit galt in dem Projekt der Entwicklung von maßgebenden Zielen zur Darstellung der Umweltleistung sowie den verschiedenen Einflussfaktoren auf die Umweltleistung in Deutschland und Brasilien.

Zur Durchführung der Untersuchung wurde ein Modell zur erweiterten Umweltleistungsbewertung entwickelt und in jeweils sechs Unternehmen der Chemie- und Textilbranche in Deutschland und Brasilien angewendet. In der Studie wurden nur Unternehmen untersucht, die schon längere Zeit ein Umweltmanagementsystem implementiert hatten und, im Bereich Chemie, Tochterunternehmen im anderen Land haben. Die Entwicklung des Modells erfolgte in Anlehnung an das Excel-

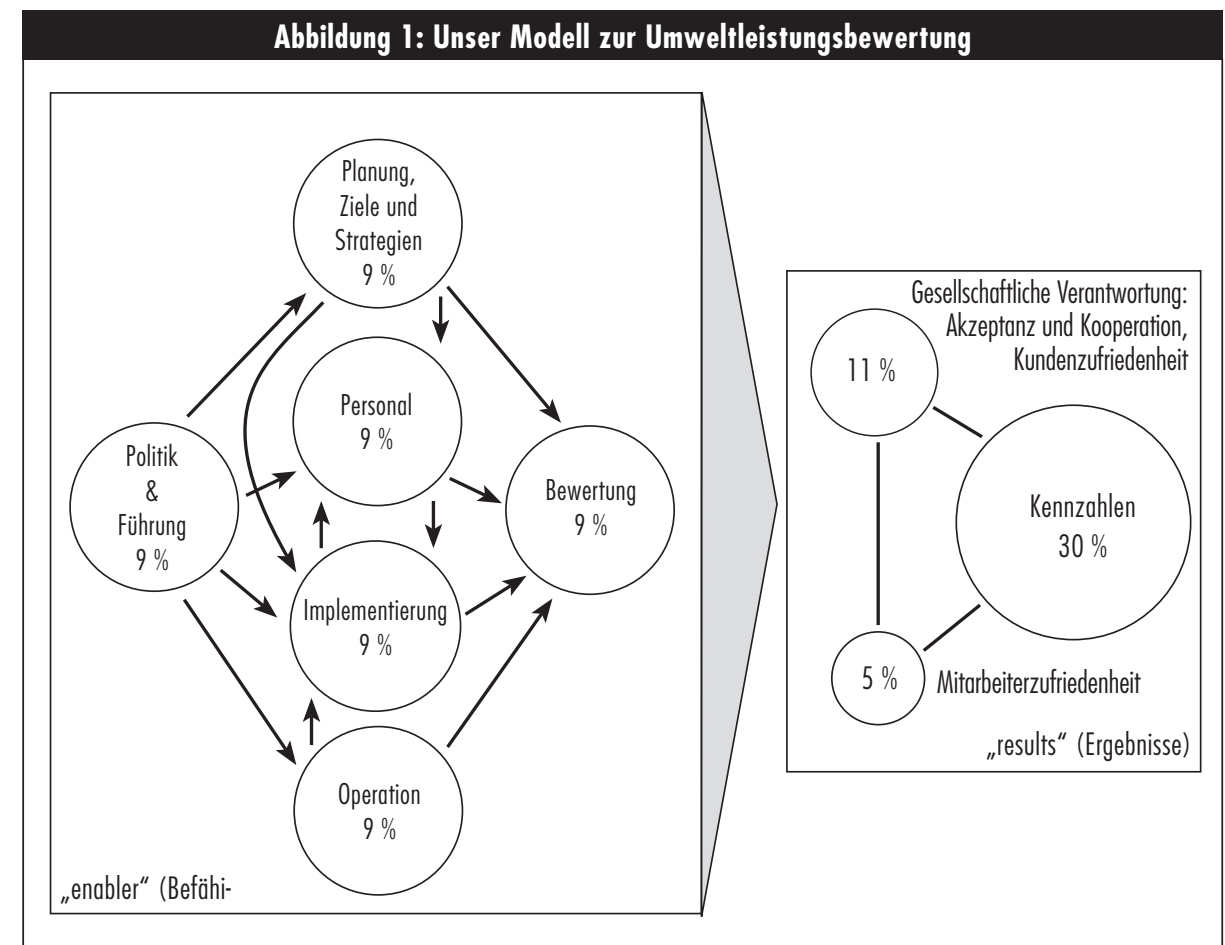

Quelle: eigene Darstellung 
lence-Modell der European Foundation for Quality Management (EFQM) und die Erweiterung des Modells durch Stahlmann/ Clausen (4). Die Umweltleistungsbewertung wurde zusätzlich durch die Abgrenzung des Untersuchungsraumes in Umwelt-, Sozial- und Visionsraum erweitert. Im Umweltraum betrachten wir alle Abläufe in den Unternehmen, die Einwirkung auf die Lieferanten und die Kunden sowie den Bereich Verkehr. Eine Untersuchung der einzelnen Produkte fand nicht statt. Bei der Untersuchung des Sozialraums orientierten wir uns an den Nachhaltigkeitszielen der Bundesregierung. Der Visionsraum soll aufzeigen, welche Ideen zur Umsetzung der Zukunftsfähigkeit in den Unternehmen entwickelt werden. Für alle Bereiche gibt es sowohl quantitative als auch qualitative Fragen und Antworten.

\section{Nachhaltigkeit in Bewertung integrieren}

Wir verstehen Umweltleistung als Erfüllung von globalen, nationalen und unternehmensspezifischen Zielen. Den Rahmen für das Globalziel bildet das Ziel einer Nachhaltigen Entwicklung. Alle weiteren, untergeordneten Ziele sollten sich daran orientieren. In dieser Definition steckt eine strategische, in die Zukunft gerichtete Komponente. Hier wird gefragt, inwiefern die Unternehmen sich schon auf dem Weg zum Nachhaltigkeitsmanagement bewegen. Die operationale Komponente umfasst die Fragen nach den Instrumenten, die die Umsetzung dieses Weges ermöglichen. Zukunftsstrategien entwickeln sich immer aus Erfahrungen aus der Vergangenheit. Wir haben deshalb untersucht, welche Entwicklung die ausgesuchten Firmen in den letzten drei Jahren gemacht haben, wie sie ihre Umweltleistung beurteilen und wie wir die Entwicklung - angesichts unserer weiter gefassten Definition - einschätzen. Aus dieser Definition wird auch ersichtlich, dass es sich um eine erweiterte Umweltleistungsbeurteilung handelt, da wir Kriterien der Nachhaltigkeit durch die globale Zielorientierung mit einbeziehen. Das Modell unterscheidet zwischen ermöglichenden Faktoren wie Personal oder Führung und ergebnisorientierten Faktoren wie Mitarbeiterzufriedenheit und bewertet diese jeweils anhand einer Punkteskala. Im Bereich der enablers sind insgesamt 540 Punkte und im Bereich der results 460 Punkte zu erreichen (vgl. Abb. 1).
Der Kennzahlenbereich der Ökoeffizienz und Ökoeffektivität umfasst davon insgesamt ein Punktespektrum von 300.

Auf der Basis unseres Modells wurden in allen Unternehmen ganztägige qualitative Interviews mit verschiedenen Personen durchgeführt. Die von uns gewählte Abgrenzung des Untersuchungsraumes machte einen Vergleich der Ergebnisse innerhalb und zwischen den Branchen möglich, was uns hinterher auch von den teilnehmenden Unternehmen bestätigt wurde. Der Vergleich der Gesamtpunktzahl zwischen Textilund Chemieunternehmen zeigt, dass insgesamt die Chemieunternehmen besser bezüglich der erweiterten Umweltleistung sind. Diese Aussage wird verstärkt, wenn wir die Ergebnisse in den Untersuchungsräumen (Umwelt-, Sozial- und Visionsraum) und zwischen den enablers und results vergleichen. So zeigte sich zum Beispiel, dass die langjährige Implementierung der Umweltmanagementsysteme in den Chemieunternehmen zu einem sehr hohen Standard bezüglich der reinen Umweltleistung geführt hat. Unterschiede werden deutlich, wenn man die Nachhaltigkeitsorientierung der Unternehmen vergleicht.

\section{Ökologisches Wirtschaften Sparen Sie sich Zeit und Geld mit dem $\mathrm{Abo}^{+}$!}

\footnotetext{
_Sie teilen sich Ökologisches Wirtschaften mit den anderen 20 Kollegen im Verteiler und bekommen sie erst Monate später in die Hand?

_Sie verbringen einige Zeit im Ausland, wollen aber keine Ausgabe von Ökologisches Wirtschaften verpassen und es pünktlich und kostengünstig erhalten?
}

Das $\mathrm{Abo}^{+}$von Ökologisches Wirtschaften kann Ihre Probleme lösen:

_Bestellen Sie zu Ihrem Print-Abo zusätzlich ein Online-Abo zum halben Preis!

_Sie erhalten die Online-Version eine Woche vor dem Erscheinen der Print-Version.

_Schluss mit der Warteschlange am Kopierer, drucken Sie sich die interessanten Artikel bequem an Ihrem Rechner aus!

*) Abo+ funktioniert nur in Kombination mit einem Print-Abo von Ökologisches Wirtschaften.

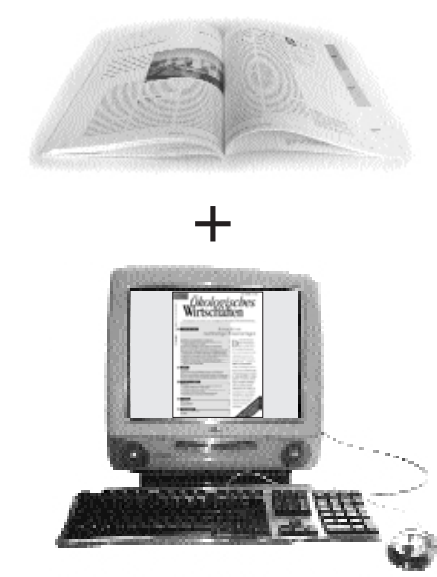

Firma, Abteilung:

Name, Vorname:

Straße, Postfach:

PLZ/Ort:

E-Mail:

Kundennr:
$\mathrm{Ja}$, ich bestelle $\mathrm{Abo}^{+}$(Print- und Online-Abo) als

○ Firmenabo für $142,50 €$

$\bigcirc$ Normalabo für $72,00 €$

OStudentenabo für $48,00 €$

Ja, ich bestelle die Online-Version zusätzlich

$\bigcirc$ für Firmen zum Aufpreis von 47,50€

$\bigcirc$ als Normalabonnent zum Aufpreis von $24,00 €$

für Studenten zum Aufpreis von 16,00€ 


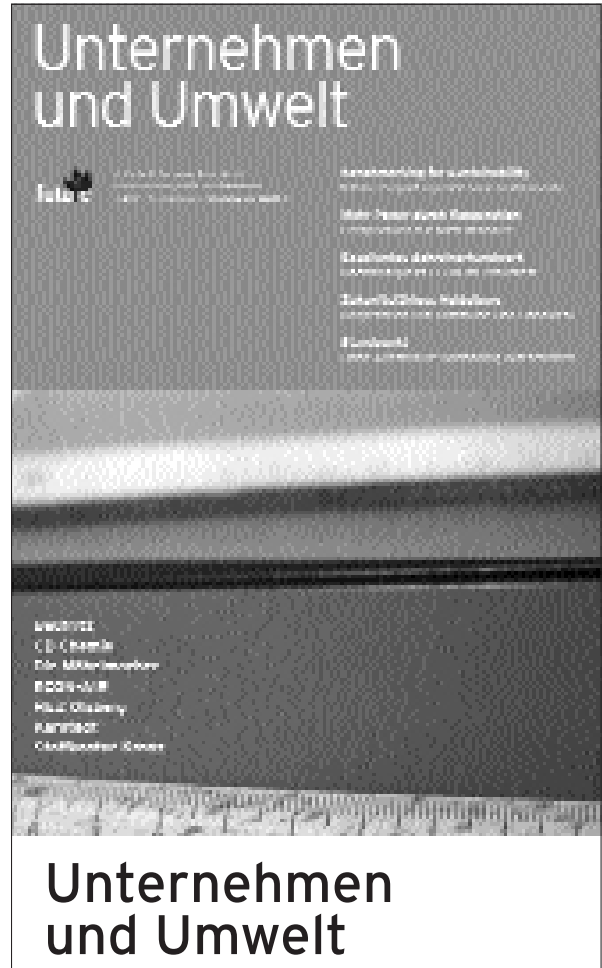

... ist die Zeitschrift für umweltorientierte Unternehmenspolitik von future e.V.

bietet Fachinformationen zum Umweltschutz und Best-Practice-Beispiele aus Unternehmen.

. bietet viermal im Jahr Unternehmensbeispiele, Literatur- und Veranstaltungshinweise.

Mit einem Schwerpunktthema in jedem Heft. Zum Beispiel:

3-4/02: Benchmarking for Sustainability 2/02: Ökomarketing

1/02: Nachhaltigkeit und Gewerbebau 4/01: Nachhaltigkeit im Handwerk

3/01: Klimaschutz

2/01: Nachhaltiges Investment

1/01: Grüne Bauwirtschaft

4/00: Kooperative Textilwirtschaft

3/00: Integrierte Produktpolitik

2/00: Soft Facts im Umweltschutz

Bestellen Sie ihr kostenloses

Probe-Abo im Internet unter

$w w w . f u t u r e-e v . d e$

future e.V. Büro Bochum

Am Varenholt 123

44797 Bochum

Fon: 0234/9799513

Fax: 0234/9799514

bochum@future-ev.de

www.future-ev.de

\section{Abbildung 2: Vergleich der Ergebnisfaktoren in den Untersuchungsräumen (Textilindustrie)}

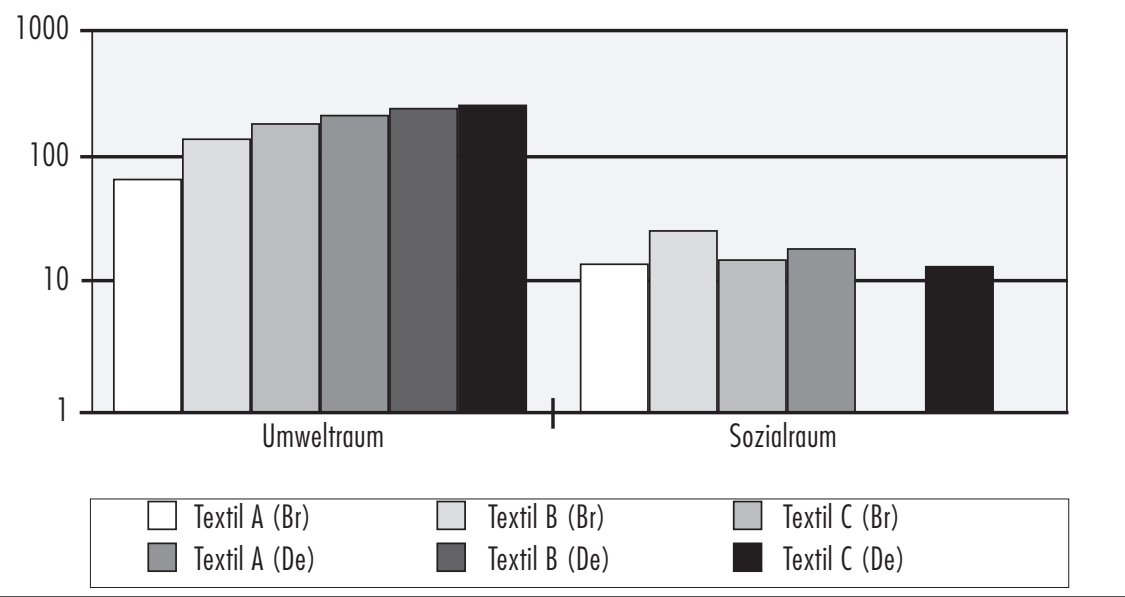

Quelle: eigene Darstellung

Ein etwas anderes Bild ergibt der Vergleich der Textilunternehmen. Hier zeigten sich mehr Unterschiede in allen Untersuchungsräumen. Noch größer werden die Unterschiede, wenn wir die Ergebnisse vergleichen, in denen die Aspekte der gesellschaftlichen Verantwortung, Kooperation und Akzeptanz sowie die Mitarbeiter- und Kundenzufriedenheit einfließen (vgl. Abb. 2).

Wir können insgesamt festhalten, dass im Umweltraum die deutschen Unternehmen nur geringe Schwankungen aufweisen und die Unternehmen beider Branchen jeweils besser als die brasilianischen Unternehmen abschnitten. Im Sozial- und Visionsraum sind größere Unterschiede festzustellen. Im Sozialraum sind brasilianische Unternehmen besser als die Deutschen. Das hat teilweise mit der langen Tradition dieser Unternehmen in den Städten zu tun. Hier werden oft ganze Familien beschäftigt und die Einwohner sind stolz auf ihr Unternehmen vor Ort. In einem brasilianischen Unternehmen gibt es beispielsweise einen Moralraum, wo persönliche Probleme der Mitarbeiter besprochen werden können. Der Firmenchef wird als Vaterfigur akzeptiert.

Als weiteren Grund für die Unterschiede kann das stärkere gesellschaftliche Interesse an Umweltthemen in Deutschland genannt werden. So gibt es in Brasilien zwar Leitbilder der Nachhaltigkeit, aber keine konkreten Umweltziele. Umweltberichte - auch die von international tätigen Unternehmen - liegen auf portugiesisch nicht vor. Die Rio-Deklaration war den brasilianischen Unternehmen weitgehend unbekannt, obgleich die ersten Interviews zur Zeit der JohannesburgKonferenz durchgeführt worden sind. Andererseits konnten wir viel gelebte Nachhaltigkeit feststellen. Standorte befinden sich in Stadtteilen von Sao Paulo, wo es einen sehr hohen Anteil an Kriminalität und Arbeitslosigkeit gibt. Die Einwohner dort als Arbeitskräfte zu beschäftigen ist gelebte Nachhaltigkeit, wie es sie in Deutschland so nicht gibt.

\section{Anmerkungen}

(1) Vgl. DIN ISO 14001; 3.8

(2) Siehe auch Richardsen, S/ Grahl, B: Anwendernutzen auch für KMU. Praxiserfahrungen mit Umweltkennzahlensystemen nach ISO 14031 in: Ökologisches Wirtschaften, 5/2002, S. 30-31

(3) Das Forschungsprojekt „Erweiterte Umweltleistungsbewertung - ein branchenspezifischer Vergleich zwischen Deutschland und Brasilien wurde vom Dezember 2001 bis zum Frühjahr dieses Jahres an der Universidade Regional Blumenau (Brasilien) und an der Fachhochschule Berlin durch durchgeführt.Die Ergebnisse erscheinen im Herbst 2003 im ökom Verlag .

(4) EFQM (Hrsg.): Das EFQM-Modell für Excellence, Brüssel, 1999; Stahlmann, Volker/ Clausen, Jens: Umweltleistung von Unternehmen - Von der Öko-Effizienz zur ÖkoEffektivität, Wiesbaden 2000, vgl. auch Stahlmann, Volker/ Clausen, Jens: Selbstbewertung mit Anspruch. In: Ökologisches Wirtschaften, 1/2001, S. 19-21.

\section{Die Autorinnen}

Anja Grothe-Senf ist Professorin an der Fachhochschule für Wirtschaft in Berlin. Beate Frank ist Professorin an der Universidade Regional de Blumenau in Brasilien.

Kontakt: Fachhochschule für Wirtschaft, Badensche Straße 50-51, 10825 Berlin. Tel. 030-85789116, E-Mail: angrothe@fhw-berlin.de 
(c) 20I0 Authors; licensee IÖW and oekom verlag. This is an article distributed under the terms of the Creative Commons Attribution Non-Commercial No Derivates License (http://creativecommons.org/licenses/by-nc-nd/3.o/), which permits unrestricted use, distribution, and reproduction in any medium, provided the original work is properly cited. 